\title{
Modelling the oscillating star V1162 Ori
}

\author{
R. Civelek, N. Kızıloğlu, and H. Kırbıyık \\ Department of Physics, Middle East Technical University, Inönü Bulvarı, 06531 Ankara, Turkey \\ Received 12 May 2003 / Accepted 12 August 2003

\begin{abstract}
Evolutionary stellar models of V1162 Ori, with the latest observations of a multiperiodic $\delta$ Scuti star, have been calculated theoretically and compared with the observations. Theoretical models representative of the star indicate that it is at an advanced stage of its main-sequence evolution. Calculations have been made for two different masses, 1.80 and $1.82 M_{\odot}$, at different rotational speeds. Rotation was treated as a perturbation and only the first-order effect was considered. The evolutionary models were used to obtain the radial and non-radial adiabatic oscillation frequencies and the oscillation equations were treated linearly. The radial fundamental frequency $\left(12.7082 \mathrm{~d}^{-1}\right)$ named so by the observers and the other observed frequencies were theoretically found from the model (model 4) that is claimed to represent the observed star the best. The agreement with the observations is within a few hundredths of a cycle or better. Since an observational rotational value of $v \sin i=46 \pm 4 \mathrm{~km} \mathrm{~s}^{-1}$ is published in the literature, rotational effects on the oscillation modes were also considered. It is shown that the oscillation frequencies increase with rotational speed for rotational velocities larger than about $50 \mathrm{~km} \mathrm{~s}^{-1}$.
\end{abstract}

Key words. stars: oscillations - stars: variables: $\delta$ Scuti - stars: individual: V1162 Ori

\section{Introduction}

$\delta$ Scuti stars are pulsating A-F variables located on or up to about 2 mag above the main-sequence. In some cases, the pulsations show variable amplitude from season to season. This is common among the multiperiodic low amplitude $\delta$ Scutitype variables $(D V<0.1 \mathrm{mag})$. The same has also been detected in a number of $\delta$ Scuti variables of medium amplitude (0.1 mag $<D V<0.3 \mathrm{mag}$ ), as in the case of V1162 Ori. The observed amplitudes range from a few thousandths of a magnitude up to about one magnitude.

The variability of V1162 Ori was discovered by Lampens (1985), and its period was found to be 0.078684 days. Poretti et al. (1990) re-observed the star, but found no other periodicity. They found a slightly increased half-amplitude. Solano \& Fernley (1997) give a value of $46 \pm 4 \mathrm{~km} \mathrm{~s}^{-1}$ for the projected rotational velocity $(v \sin i)$ of the star.

Hintz et al. (1998) from new observations obtained values for the mean effective temperature of the star of $7540 \pm 100 \mathrm{~K}$, for the mean surface gravity of $\langle\log g>=3.96 \pm 0.07$ and for the bolometric absolute magnitude of 2.0 mag. By using the evolutionary tracks of Schaerer et al. (1993) they estimated a mass of $1.84 M_{\odot}$ and an age of $0.6 \mathrm{Gyr}$ for V1162 Ori. A second possible periodicity was detected $\left(16.4864 \mathrm{~d}^{-1}\right)$, but it was later shown that it arose from a variable comparison star (Lampens \& Van Cauteren 2000).

Arentoft \& Sterken (2000) observed the star and found some amplitude changes. In fact, $\delta$ Scuti stars show amplitude

Send offprint requests to: $\mathrm{R}$. Civelek,

e-mail: rikkat@photon.physics.metu.edu.tr variations on time scales of from days to years (Breger 2000). Arentoft et al. (2001) organized a multisite campaign and observed the star at 15 different observatories around the world, from October 1999 to May 2000, to further investigate amplitude and period variability on short time scales. They found that the star was multiperiodic and $12.7082 \mathrm{~d}^{-1}$ was the dominating frequency. All frequencies obtained from their multisite observations are listed in Table 3 of their paper (cf. Table 1). Among the 6 observed frequencies, $f_{5}\left(=15.9901 \mathrm{~d}^{-1}\right)$ was noted to be an alias and $f_{6}\left(=27.7744 \mathrm{~d}^{-1}\right)$ might be uncertain due to the very low amplitude. Besides the main dominating frequency 5 additional frequencies in the amplitude spectrum were found $\left(f_{2}, f_{3}, f_{4}, f_{5}\right.$, and $\left.f_{6}\right)$. Looking at their period ratios and assuming that $f_{1}$ is radial in the fundamental mode, it was argued that they could be nonradial oscillation frequencies. They assigned the values $T_{\mathrm{e}}=7400 \mathrm{~K}$ and $M_{v}=1.89$ to V1162 Ori. Assuming a mass of $1.80 M_{\odot}$ and a bolometric correction of -0 . 1 , the $Q$-value for the dominating frequency was found to be $0.029 \mathrm{~d}$. Although the $Q$-value does not agree very well with the fundamental mode for which $Q=0.033$, they assumed the dominant frequency $12.708 \mathrm{~d}^{-1}$ as the fundamental mode of radial pulsation.

\section{The models}

The stellar evolutionary code used in this study is described in Yıldız \& Kızıloğlu (1997), which is a modified version of Ezer's stellar evolution code (Ezer \& Cameron 1967). OPAL opacity tables (Iglesias et al. 1992) and the MHD equation of state were applied to the stellar models (Mihalas et al. 1990). 


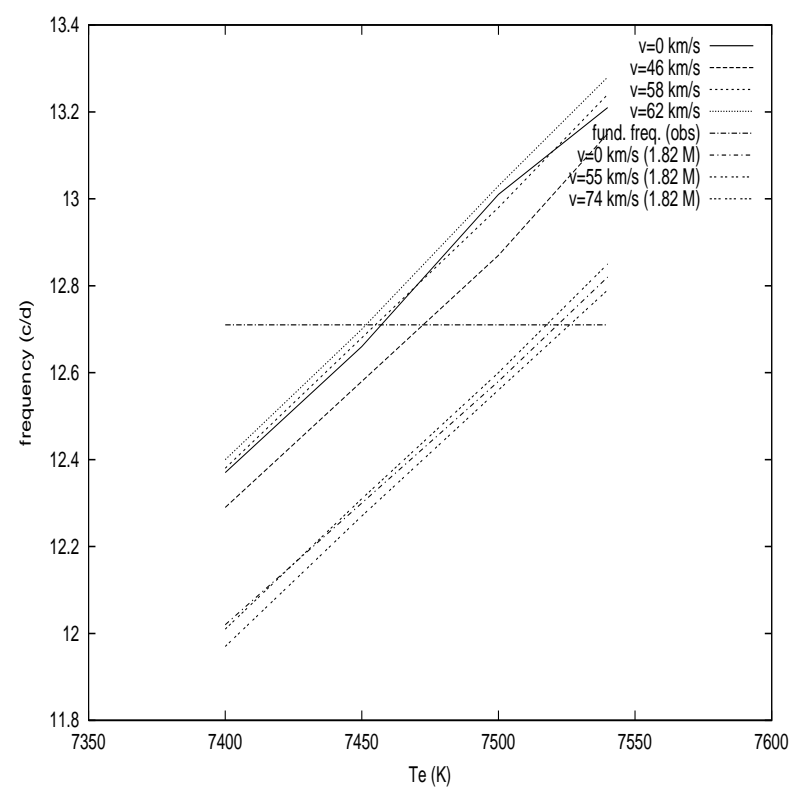

Fig. 1. Frequency versus effective temperature showing the effective temperature intervals corresponding to the main observed frequency $12.708 \mathrm{~d}^{-1}$ for the two mass models of 1.80 and $1.82 M_{\odot}$ with different rotation velocities.

Table 1. The observed frequencies of V1162 Ori.

\begin{tabular}{lllll}
\hline \hline ID & $\begin{array}{l}\text { Frequency } \\
\mathrm{d}^{-1}\end{array}$ & $\begin{array}{l}\text { Amplitude } \\
\mathrm{mmag}\end{array}$ & $S / N$ & $f_{1} / f_{\mathrm{n}}$ \\
\hline & & & & \\
$\mathrm{f}_{1}$ & 12.7082 & 66.6 & 151.0 & 1.000 \\
$2 \mathrm{f}_{1}$ & 25.4164 & 6.6 & 27.0 & \\
$3 \mathrm{f}_{1}$ & 38.1246 & 1.3 & 8.0 & \\
$\mathrm{f}_{2}$ & 12.9412 & 3.2 & 7.0 & 0.982 \\
$\mathrm{f}_{3}$ & 19.1701 & 3.0 & 9.0 & 0.663 \\
$\mathrm{f}_{4}$ & 21.7186 & 2.4 & 8.0 & 0.585 \\
$\mathrm{f}_{5}$ & 15.9901 & 2.1 & 5.5 & 0.795 \\
$\mathrm{f}_{6}$ & 27.7744 & 1.1 & 5.0 & 0.458 \\
& & & & \\
\hline
\end{tabular}

To model V1162 Ori, we computed a series of evolutionary sequences for masses 1.80 and $1.82 M_{\odot}$. We used an initial solar composition of $(X, Z)=(0.7,0.02)$ and a mixing length parameter $\alpha=1.75$. Models were evolved with no rotation and with uniform rotation. In the evolutionary calculations of 1.80 and $1.82 M_{\odot}$ stars, different equatorial rotation velocities were considered.

The models were evolved up to the point where the luminosity and the effective temperature almost satisfy the observed values in V1162 Ori.

Figure 1 shows the variation of frequency of some models with different equatorial rotation velocities, with effective temperature. We took the frequency, $12.7082 \mathrm{~d}^{-1}$, as the radial fundamental frequency of V1162 Ori as suggested by Arentoft et al. (2001). It is clear from Fig. 1 that the radial fundamental frequency matches the combination of the masses used in the modeling and the values for $T_{\text {eff }}$ given by Hintz et al. (1998) and Arentoft et al. (2001). The calculations were done for
$T_{\text {eff }}=7400 \mathrm{~K}, 7450 \mathrm{~K}, 7500 \mathrm{~K}$ and $7540 \mathrm{~K}$ for different values of the equatorial velocity, and with the condition that the luminosity should remain within the range of the observations.

Table 2 presents those models that were chosen for the close agreement between the calculated and the observed frequencies. The ages of all models range from $7.5 \times 10^{8}$ to $8.5 \times 10^{8}$ years. The hydrogen content of the core, in all models, is about $X=0.30$. The best fit to the observed parameters of V1162 Ori was given by Model 4.

\section{Radial and non-radial adiabatic oscillations}

The basic equations governing linear adiabatic oscillations are given by Cox (1980), who gives both a radial and a non-radial treatment.

For the models under consideration we adopted Eqs. (1)(3) in the paper by Al-Murad \& Kirbiyık (1995). The oscillation equations can be solved by the perturbation method if the effect of rotation is considered to be small, that is, $\Omega<\sigma$ where $\Omega$ is the angular frequency of rotation. Recalling that the smallest observed frequency is $12.7082 \mathrm{~d}^{-1}$, the perturbation method may be thought to be applicable, that is, the rotation frequency is less than the fundamental frequency by a factor of approximately 20. The eigenfunctions are expanded into powers of the angular frequency of rotation, $\Omega$ (Simon 1969). When it is observed from an inertial reference frame, the frequency of a nonradial oscillation, to the first order in $\Omega$ in a uniformly rotating star, is modified as (Ledoux \& Walraven 1958)

$\sigma_{n l m}=\sigma_{n l, 0}-m \Omega\left(1-C_{n l}\right)$,

where $\mathrm{n}$ denotes the radial order, $l$, the angular degree and $m$ the azimuthal order. $\sigma_{n l o}$ is the oscillation frequency when there is no rotation, i.e., when $m=0 . C_{n l}$ is a function calculated in terms of the nonperturbed quantities.

The procedure of solving the system of equations and the method of distinguishing $p$ and $g$ modes using the propagation diagram, $\omega^{2}$ versus $r / R$ diagram, were given in Civelek et al. (2001). Looking at the propagation diagrams given in Figs. 2 and 3 for a non-rotating star with $M_{\odot}=1.80$, we note that the increase in the number of $g$-nodes is more emphasized as the star evolves and that they are concentrated toward the central region. Hence it is expected that gravity waves are trapped inside the deep interior.

\section{Results and discussion}

The models given in Table 2 were investigated for radial and nonradial oscillations. Those frequencies that are close to the observed ones have been selected and given in Tables 3 and 4 . Note that there are two different masses, but with different rotation speeds making 7 different models whose characteristics are given in Table 2. In Tables 3 and 4 we list the calculated radial and nonradial oscillation frequencies related to the selected models. Observed frequencies close to the calculated ones are also given for the sake of comparison.

Looking at the calculated frequencies obtained in our models it seems that there are two models that may represent V1162 Ori. One of them is model 4 with $M=1.80 M_{\odot}$ and 
Table 2. The characteristics of nonrotating and uniformly rotating models with masses $1.80 M_{\odot}$ and $1.82 M_{\odot}$ compatible approximately with the characteristics of V1162 Ori. The symbols have their usual meanings.

\begin{tabular}{llllllll}
\hline \hline $\begin{array}{l}\text { Model } \\
\text { No. }\end{array}$ & $\begin{array}{l}M \\
\left(M_{\odot}\right)\end{array}$ & $\begin{array}{l}L \\
\left(L_{\odot}\right)\end{array}$ & $\begin{array}{l}R \\
\left(R_{\odot}\right)\end{array}$ & $\begin{array}{l}T_{\mathrm{e}} \\
(\mathrm{K})\end{array}$ & $\begin{array}{l}\rho_{\mathrm{c}} /<\rho>10^{2} \\
\times\end{array}$ & $\begin{array}{l}\text { Rot. Vel. } \\
\left(\mathrm{km} \mathrm{s}^{-1}\right)\end{array}$ & $\begin{array}{l}f_{\mathrm{r}, \text { fund }} \\
\mathrm{d}^{-1}\end{array}$ \\
\hline 1 & 1.80 & 13.41 & 2.201 & 7450 & 3.47 & 0.0 & 12.661 \\
2 & 1.80 & 13.49 & 2.208 & 7450 & 3.46 & 46.0 & 12.576 \\
3 & 1.80 & 13.38 & 2.195 & 7450 & 3.49 & 57.8 & 12.667 \\
4 & 1.80 & 13.28 & 2.191 & 7450 & 3.39 & 61.9 & 12.704 \\
5 & 1.82 & 13.94 & 2.190 & 7540 & 3.47 & 0.0 & 12.820 \\
6 & 1.82 & 13.95 & 2.192 & 7540 & 3.50 & 55.4 & 12.785 \\
7 & 1.82 & 13.88 & 2.210 & 7500 & 3.42 & 73.6 & 12.603 \\
\hline
\end{tabular}

Table 3. Oscillation frequencies for $1.80 M_{\odot}$ models.

\begin{tabular}{|c|c|c|c|c|c|c|c|c|}
\hline Model & $V_{\text {rot }}$ & $l$ & $m$ & $N_{\mathrm{p}}$ & $N_{\mathrm{g}}$ & $f_{\mathrm{c}}\left(\mathrm{d}^{-1}\right)$ & $f_{\text {obs }}\left(\mathrm{d}^{-1}\right)$ & $\left|f_{\mathrm{o}}-f_{\mathrm{c}}\right|$ \\
\hline \multirow[t]{3}{*}{1} & $0.0 \mathrm{~km} \mathrm{~s}^{-1}$ & 0 & 0 & 0 & 0 & 12.661 & 12.708 & 0.047 \\
\hline & & 1 & 0 & 0 & 0 & 12.959 & 12.941 & 0.018 \\
\hline & & 2 & 0 & 0 & 1 & 12.723 & 12.708 & 0.015 \\
\hline \multirow[t]{7}{*}{2} & $46.0 \mathrm{~km} \mathrm{~s}^{-1}$ & 0 & 0 & 0 & 0 & 12.576 & 12.708 & 0.132 \\
\hline & & 1 & 0 & 0 & 0 & 12.910 & 12.941 & 0.031 \\
\hline & & 1 & -1 & 1 & 0 & 16.080 & 15.990 & 0.090 \\
\hline & & 2 & 0 & 0 & 1 & 12.852 & 12.941 & 0.089 \\
\hline & & 2 & 2 & 1 & 1 & 15.905 & 15.990 & 0.085 \\
\hline & & 3 & -1 & 2 & 0 & 19.130 & 19.170 & 0.040 \\
\hline & & 3 & 1 & 2 & 0 & 21.570 & 21.719 & 0.149 \\
\hline \multirow[t]{10}{*}{3} & $57.8 \mathrm{~km} \mathrm{~s}^{-1}$ & 0 & 0 & 0 & 0 & 12.667 & 12.708 & 0.031 \\
\hline & & 1 & 0 & 0 & 0 & 12.972 & 12.941 & 0.031 \\
\hline & & 1 & -1 & 1 & 0 & 16.052 & 15.990 & 0.062 \\
\hline & & 2 & 0 & 0 & 1 & 12.757 & 12.708 & 0.049 \\
\hline & & 2 & 2 & 1 & 1 & 15.934 & 15.990 & 0.056 \\
\hline & & 2 & 0 & 4 & 0 & 27.683 & 27.774 & 0.091 \\
\hline & & 3 & 1 & 0 & 2 & 12.784 & 12.708 & 0.076 \\
\hline & & 3 & -1 & 2 & 0 & 18.944 & 19.170 & 0.226 \\
\hline & & 3 & 1 & 2 & 0 & 21.675 & 21.719 & 0.044 \\
\hline & & 3 & 1 & 3 & 0 & 25.256 & 25.416 & 0.160 \\
\hline \multirow[t]{7}{*}{4} & $61.9 \mathrm{~km} \mathrm{~s}^{-1}$ & 0 & 0 & 0 & 0 & 12.704 & 12.708 & 0.004 \\
\hline & & 1 & 0 & 0 & 0 & 12.997 & 12.941 & 0.056 \\
\hline & & 1 & -1 & 1 & 0 & 16.045 & 15.990 & 0.055 \\
\hline & & 2 & 2 & 1 & 1 & 16.016 & 15.990 & 0.026 \\
\hline & & 3 & 1 & 2 & 0 & 21.741 & 21.719 & 0.022 \\
\hline & & 3 & -1 & 2 & 1 & 18.926 & 19.170 & 0.244 \\
\hline & & 3 & 1 & 3 & 0 & 25.337 & 25.416 & 0.080 \\
\hline
\end{tabular}

$V_{\text {rot }}=61.9 \mathrm{~km} \mathrm{~s}^{-1}$ and the other is model 6 with $M=1.82 M_{\odot}$ and $V_{\text {rot }}=55.4 \mathrm{~km} \mathrm{~s}^{-1}$. Taking as criteria the radial fundamental frequency $f_{1}$, model 4 is preferred as the best model for V1162 Ori because, in this model, the agreement between the observed and calculated frequency for $f_{1}$ is within only a few thousandths of a cycle. For the second best model (model 6), the separation for $f_{1}$ is $0.027 \mathrm{~d}^{-1}$. Although in model 6 the agreement for $f_{5}$ and $f_{6}$ is better than in model 4 , these low amplitude frequencies, especially $f_{5}$, suffer from uncertainty due to aliasing as discussed by Arentoft et al. (2001), so they may not be suitable for deciding which model is better. Some additional parameters related to the model that represents the data best are given in Table 5. The frequencies 16.016 and $18.926 \mathrm{~d}^{-1}$ given in model 4 of Table 3 which were obtained for $l=2, m=2$ and $l=3, m=-1$ respectively, are mixed modes. Observation of a mixed mode is possible if there is a bumping effect as was explained by Aizenman et al. (1977). The other frequencies in model 4 of the table are all pure $\mathrm{p}$ type modes.

We will need to consider the effect of rotation in some detail. The effect has been discussed by Pamyatnykh (2000) in great detail. Assuming that model 4 represents V1162 Ori, the rotation velocity $V_{\text {rot }}=61.9 \mathrm{~km} \mathrm{~s}^{-1}$ gives an inclination angle in the range $\approx 43-54 \mathrm{deg}$. However, in the second best model with $M=1.82 M_{\odot}$ and $V_{\text {rot }}=55.4 \mathrm{~km} \mathrm{~s}^{-1}$ one gets an inclination angle in the range $\approx 49-64 \mathrm{deg}$. Thus, in both models $i>45$ degrees. This is slightly closer to equatorial than to pole-on view. Using the relation $f_{\text {rot }}=V_{\text {rot }} \sin i / 2 \pi R \sin i$ the rotational frequency is found to be around $0.56 \mathrm{~d}^{-1}$. Thus the 
Table 4. Oscillation frequencies for $1.82 M_{\odot}$ models.

\begin{tabular}{lllllllll}
\hline \hline Model & $V_{\text {rot }}$ & $l$ & $m$ & $N_{\mathrm{p}}$ & $N_{\mathrm{g}}$ & $f_{\mathrm{c}}\left(\mathrm{d}^{-1}\right)$ & $f_{\text {obs }}\left(\mathrm{d}^{-1}\right)$ & $\left|f_{\mathrm{o}}-f_{\mathrm{c}}\right|$ \\
\hline $\mathbf{5}$ & $\mathbf{0 . 0} \mathbf{~ k m ~ s}^{-1}$ & 0 & 0 & 0 & 0 & 12.820 & $\mathbf{1 2 . 7 0 8}$ & 0.112 \\
& & 3 & 0 & 0 & 1 & 12.851 & $\mathbf{1 2 . 9 4 1}$ & 0.090 \\
\hline $\mathbf{6}$ & $\mathbf{5 5 . 4} \mathbf{~ k m ~ s}^{-1}$ & 0 & 0 & 0 & 0 & 12.735 & $\mathbf{1 2 . 7 0 8}$ & 0.027 \\
& & -1 & 0 & 0 & 12.614 & $\mathbf{1 2 . 7 0 8}$ & 0.094 \\
& & 0 & 0 & 1 & 13.002 & $\mathbf{1 2 . 9 4 1}$ & 0.061 \\
& 2 & 2 & 2 & 0 & 21.738 & $\mathbf{2 1 . 7 1 9}$ & 0.019 \\
& 3 & -1 & 1 & 1 & 16.006 & $\mathbf{1 5 . 9 9 0}$ & 0.016 \\
& 3 & -1 & 2 & 0 & 19.197 & $\mathbf{1 9 . 1 7 0}$ & 0.027 \\
& 3 & -3 & 4 & 0 & 27.829 & $\mathbf{2 7 . 7 7 4}$ & 0.055 \\
& 3 & 1 & 3 & 0 & 25.440 & $\mathbf{2 5 . 4 1 6}$ & 0.024 \\
\hline $\mathbf{7}$ & $\mathbf{7 3 . 6} \mathbf{~ k m ~ s}^{-1}$ & 0 & 0 & 0 & 12.603 & $\mathbf{1 2 . 7 0 8}$ & 0.106 \\
& 1 & 0 & 0 & 0 & 12.922 & $\mathbf{1 2 . 9 4 1}$ & 0.019 \\
& 2 & -2 & 2 & 0 & 19.210 & $\mathbf{1 9 . 1 7 0}$ & 0.040 \\
& 3 & 3 & 0 & 3 & 12.792 & $\mathbf{1 2 . 7 0 8}$ & 0.084 \\
& 3 & 1 & 2 & 0 & 21.778 & $\mathbf{2 1 . 7 1 9}$ & 0.059 \\
& 3 & 2 & 1 & 2 & 15.883 & $\mathbf{1 5 . 9 9 0}$ & 0.107 \\
\hline
\end{tabular}

Table 5. Pulsation characteristics of the model 4, in radial oscillation $(l=0)$, with $M=1.80 M_{\odot}, V_{\text {rot }}=61.9 \mathrm{~km} \mathrm{~s}^{-1}$ and $T_{\mathrm{e}}=7450 \mathrm{~K}(\mathrm{model} 4$ in Table 2).

\begin{tabular}{|c|c|c|c|c|c|}
\hline $\begin{array}{l}f_{\mathrm{c}} \\
\left(\mathrm{d}^{-1}\right) \\
\end{array}$ & $\begin{array}{l}Q \times 100 \\
\text { (days) }\end{array}$ & $Q / Q_{\mathrm{F}}$ & Mode & $\begin{array}{l}f_{\text {obs }} \\
\text { (Arentoft et al.) } \\
\left(\mathrm{d}^{-1}\right)\end{array}$ & $\begin{array}{l}f_{\text {obs }} \\
\text { (Hintz et al.) } \\
\left(\mathrm{d}^{-1}\right) \\
\end{array}$ \\
\hline 12.704 & 3.25 & - & $\mathrm{F}$ & 12.708 & 12.709 \\
\hline 16.492 & 2.50 & 0.77 & 10 & - & 16.486 \\
\hline 20.610 & 2.00 & 0.62 & 20 & - & - \\
\hline
\end{tabular}

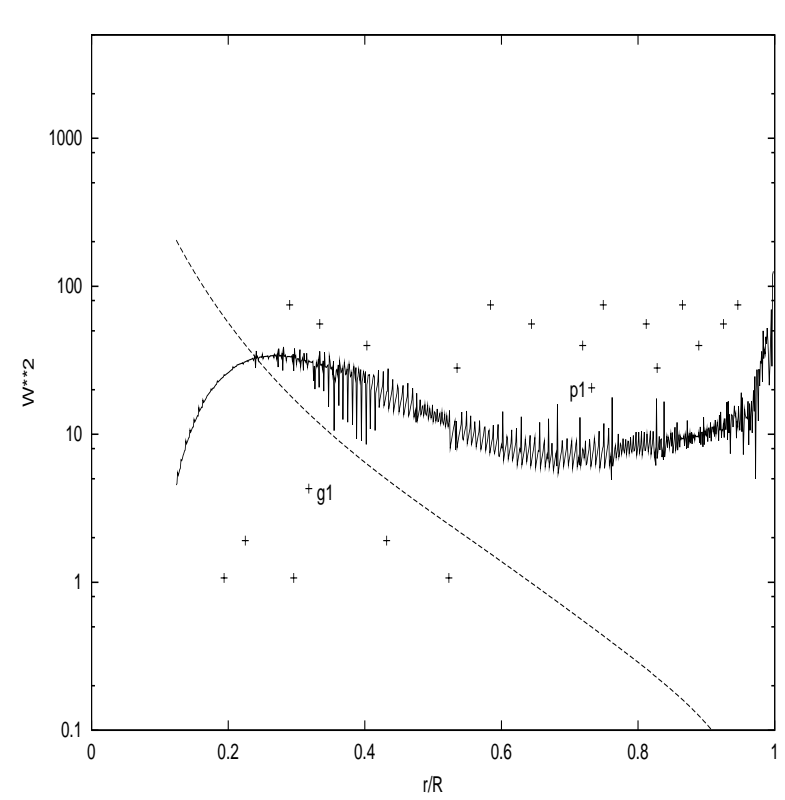

Fig. 2. Propagation diagram plotted for $N^{2}$ (Brunt Vaisala frequency) and $L_{1}^{2}$ (Lamb frequency, $l=1$ ) throughout the star; showing $p$ and $g$ modes for a nonrotating model of $1.80 M_{\odot}$ with $X=0.68$.

contribution of the rotational frequency to the oscillation frequencies is expected to be of that order. Keeping in mind the fact that rotation will deform a star and change its structure,

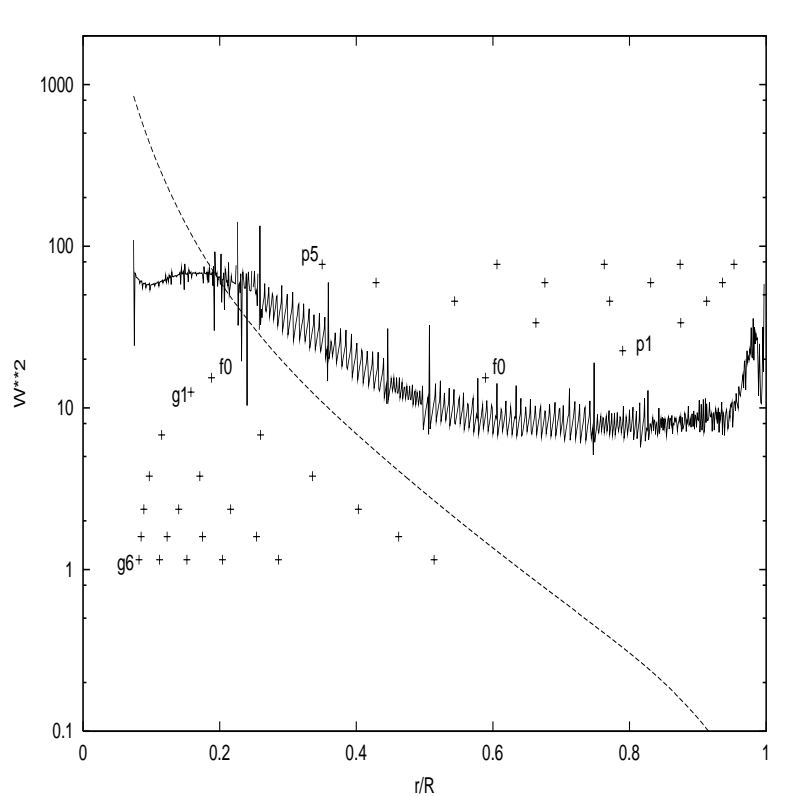

Fig. 3. Propagation diagram plotted for $N^{2}$ (Brunt Vaisala frequency) and $L_{1}^{2}$ (Lamb frequency, $l=1$ ) throughout the star; showing $p$ and $g$ modes for a nonrotating model of $1.80 M_{\odot}$ with $X=0.10$. A more evolved model compared to the one in Fig. 2.

we have calculated models with different rotational speeds. Figure 4 shows the variation of the oscillation frequencies near 


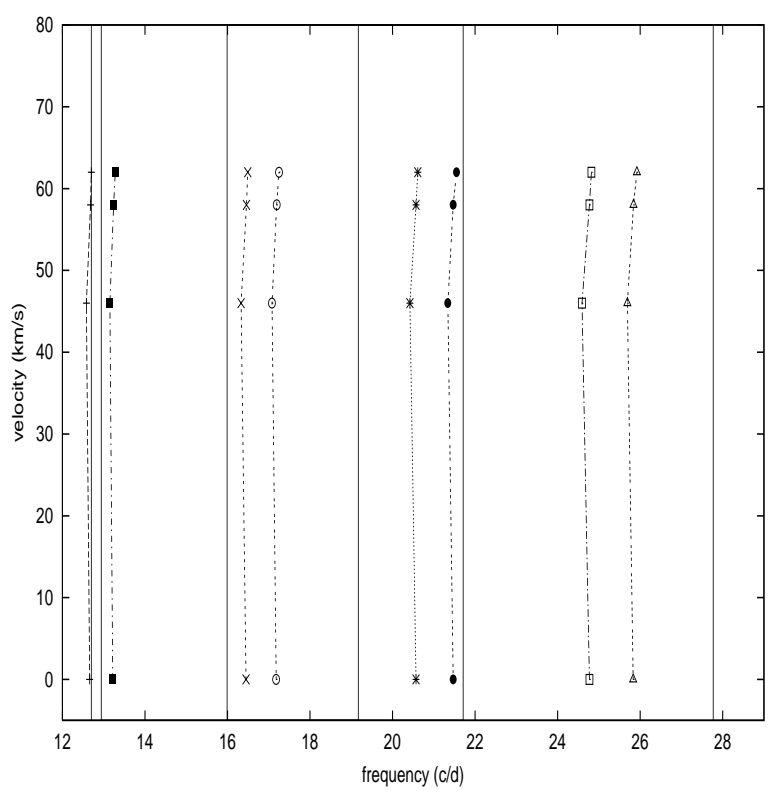

Fig. 4. Variation of the radial fundamental frequency, first, second and third overtone frequencies (dotted lines) with different rotation velocities for $1.80 M_{\odot}$ star model in the vicinity of the observed frequency (solid line) of V1162 Ori. The variations are given for two different effective temperature values of $7450 \mathrm{~K}$ and $7540 \mathrm{~K}$, at each frequency.

the observed ones for a spherical harmonic degree $l=0$ for a $1.8 M_{\odot}$ star and for two different effective temperatures. Figure 4 confirms the finding of Pamyatnykh (2000, Fig. 12) that as the velocity of the model star increases, changes in frequency are seen for a rotational velocity greater than about $50 \mathrm{~km} \mathrm{~s}^{-1}$. However, in the present work, the frequency corresponding to rotational velocity $46 \mathrm{~km} \mathrm{~s}^{-1}$ is lower than the frequency corresponding to zero velocity. This is not observed in Fig. 12 of Pamyatnykh (2000). We did not consider the terms other than the first order effect in rotation while Pamyatnykh (2000) included the effect of rotation on the oscillation frequencies up to the third order in rotation. The additional terms in his work might have shifted frequencies in the rotating stars (Dziembowski \& Goode 1992).

To see whether we can obtain the observed $12.708 \mathrm{~d}^{-1}$ frequency as a first overtone frequency rather than the fundamental radial frequency, we also tried nonrotating star models having masses greater than $1.82 M_{\odot}$ for radial oscillations. A relevant frequency to V1162 Ori was found only in the $2 M_{\odot}$ model. The calculated radial fundamental frequency was found to be $9.851 \mathrm{~d}^{-1}$ while the first overtone frequency was obtained to be $12.691 \mathrm{~d}^{-1}$ which is close to the observed frequency of V1162 Ori $\left(12.708 \mathrm{~d}^{-1}\right)$ within an accuracy of few parts in $10^{2}$. The effective temperature for the model is $7570 \mathrm{~K}$, while the luminosity is $21.30 L_{\odot}$. Note that the effective temperature is within the range of the quoted values in literature, but the luminosity is a bit larger than that for the models with 1.80 and 1.82 solar masses. If a 2 solar mass model is taken as possibly representative of the star, it seems that the observed frequency $12.708 \mathrm{~d}^{-1}$ is not the radial fundamental frequency but the first overtone frequency. For the $12.708 \mathrm{~d}^{-1}$ frequency to be the first overtone frequency, the observed luminosity of this star must be greater than the present determined value.

From our results, we suggest that the $\delta$ Scuti star V1162 Ori may have a mass in the range from 1.80 to $1.82 M_{\odot}$ with rotation speed in the range from $55.4 \mathrm{~km} \mathrm{~s}^{-1}$ to $61.9 \mathrm{~km} \mathrm{~s}^{-1}$.

\section{References}

Aizenman, M., Smeyers, P., \& Weigert, A. 1977, A\&A, 58, 41

Al-Murad, M. A., \& Kirbıyık, H. 1995, in Proc. 32nd Liege Int. Astrop. Coll., Stellar Evolution: What should be done, ed. A. Noels, et al., p. 389

Arentoft, T., \& Sterken, C. 2000, A\&A, 354, 589

Arentoft, T., Sterken, C., Handler, G., et al. 2001, A\&A, 374, 1056

Breger, M. 2000, MNRAS, 313, 129

Civelek, R., Kızıloğlu, N., \& Kırbıyık, H. 2001, AJ, 122, 2042

Cox, J. P. 1980, Theory of Stellar Pulsation, Princeton University

Dziembowski, W. A., \& Goode, P. R. 1992 , ApJ, 394, 670

Ezer, D., \& Cameron, A. G. W. 1967, Can. J. Phys., 45, 3461

Hintz, E. G., Joner, M. D., \& Kim, C. 1998, PASP, 110, 689

Iglesias, C. A., Rogers, F. J., \& Wilson, B. G. 1992, ApJ, 397, 717

Lampens, P. 1985, IBVS, 2794

Lampens, P., \& Van Cauteren, P. 2000, IBVS, 4857

Ledoux, P., \& Walraven, T. H. 1958, Handb. Der. Phys. 51, ed. S. Flugge, 353

Mihalas, D., Hummer, D. G., Mihalas, B. W., \& Dappen, W. 1990, ApJ, 350, 300

Pamyatnykh, A. A. 2000, in Delta Scuti and Related Stars, ed. M. Breger, \& H. Montgomery, ASP Conf. Ser., 210, 215

Poretti, E., Antonello, E., \& Le Borgne, J. F. 1990, A\&A, 228, 350

Schaerer, D., Charbonnel, C., Meynet, G., Maeder, A., \& Schaller, G. 1993, A\&AS, 102, 339

Simon, R. 1969, A\&A, 2, 390

Solano, E., \& Fernley, J. 1997, A\&AS, 122, 131

Yıldız, M., \& Kızıloğlu, N. 1997, A\&A, 326, 187 